\section{Antidepressant use and the risk of epilepsy and seizures in people aged 20 to 64 years: A cohort study using a primary care database (1)}

Evidence suggests that antidepressant (ADTs) use may increase the risk of seizures. In the UK about one percent of the population is affected with epilepsy. Since the use of ADTs is on the rise in the UK, the authors of this study aimed to assess and quantify the change in absolute risk of epilepsy or seizures associated with antidepressant use. Patients on antidepressants who were aged between 20-64 years, who did not have known predisposition to epilepsy and who had no past history of seizures were included.

Patient data from 687 general practices across the UK was extracted from the QResearch primary care database. In the UK, depression is diagnosed most often by general practitioners. Participants were selected for the study if they were diagnosed with a first episode of depression between the ages of 20 to 64 years. Patients with bipolar affective disorder, schizophrenia or other psychotic illnesses or who were on lithium or anti-manic medications, patients who had a diagnosis of depression or who were prescribed ADTs before the study entry date or before the age of 20 were excluded from the study. Follow-up was continued until the patient's first recorded diagnosis of epilepsy or seizures, or until the patient left the practice, death, or the end of the study period.

ADTs were classified into tricyclic and related antidepressants (TCAs), selective serotonin reuptake inhibitors (SSRIs), monoamine oxidase inhibitors (MAOIs), and other antidepressants.

Both demographic and baseline characteristics and comorbidities were included as confounding factors in the adjusted analyses. Demographic features included were age at study entry, gender, year of index diagnosis of depression, severity of index diagnosis of depression, Townsend deprivation score, smoking status and severity, alcohol intake and severity, and ethnicity. The authors adjusted for 13 baseline comorbidities and for usage of 11 drugs at baseline.

Cox's proportional hazards models were used to analyse the time to the first recorded diagnosis of epilepsy or seizures and to assess any differences in risk associated with the antidepressant prescribed.
The initial cohort consisted of 327,235 patients aged 20 to 64 years with a first diagnosis of depression recorded during the study period, but only 238,963 were included in the analysis ( $73 \%$ of the initial sample). There were 146,028 (61.1\%) females and 92,935 (38.9\%) males, with a mean age of 39.5 years $(\mathrm{SD}=11.1)$.

Asthma and/or chronic obstructive airways disease $(31,816$ patients, $13.3 \%)$ was the most common comorbidity at baseline followed by hypertension $(17,217$ patients, $7.2 \%$ ). The most commonly prescribed medication at baseline was anti-hypertensives $(25,344$ patients, $10.6 \%$ ).

During the first 5 years of follow-up 878 patients (416 women and 462 men) developed epilepsy or seizures, giving an incidence rate of 99 per 100,000 person-years (76 in women and 135 in men). All hazard ratios for the different antidepressant drug classes were statistically significant compared with periods of no use, with the highest being for combined antidepressants (adjusted HR 2.73, 95\% CI 1.52 to 4.91). Trazodone (adjusted HR $=5.41,95 \%$ CI 3.05 to 9.61), lofepramine (adjusted $\mathrm{HR}=3.09,95 \% \mathrm{CI} 1.73$ to 5.50 ), and venlafaxine (adjusted HR $=2.84,95 \%$ CI 1.97 to 4.08 ) were associated with highest risk of seizures/epilepsy. Escitalopram, sertraline and mirtazapine, were not associated with a significant increase in risk.

The authors concluded that since there is an increase risk of seizures or epilepsy in patients treated with ADT, the use of ADT needs to be weighed against the potential benefits of treatment. Further the duration of treatment needed should also be considered, as the risk seems to accumulate with long-term treatment. The authors also emphasized the need for further studies in this important area, since the studies so far report conflicting conclusions.

\section{What are the similarities and differences between schizophrenia and schizophrenia- like psychosis of epilepsy? A neuropatho- logical approach to the understanding of schizophrenia spectrum and epilepsy (2)}

Patients with temporal lobe epilepsy (TLE), have an increased risk of psychosis. In this review, the authors summarize key clinical and neuro-pathological features, 
comparing schizophrenia and schizophrenia-like psychosis in TLE.

Since its first description by Kraepelin as dementia praecox, schizophrenia $(\mathrm{SCH})$ has been studied extensively, yet its precise clinical nature remains undefined. But current evidence suggests that it is a constellation of conditions rather than a single entity. The core features include positive, negative, cognitive, disorganization, mood and motor symptoms. The manifestation of symptoms varies among patients and along the course of the illness.

In DSM 5, psychotic disorders are categorized under 'schizophrenia spectrum and other psychotic disorders' and the subtyping of $\mathrm{SCH}$ is removed. A crucial difference described between $\mathrm{SCH}$ and other psychotic disorders is the decrease in the level of functioning (below premorbid levels) seen in SCH. Variable degrees of association and expression of these symptom dimensions may reflect distinct pathogenic processes associated with distinct risk factors, and possibly, differences in prognosis, treatment, and pathology.

Interictal psychosis (IP) of TLE, closely resemble $\mathrm{SCH}$, but in the former, the patients tend to have more religious themes and paranoid ideas, the affect tends to remain warm and appropriate and without typical deterioration into hebephrenic state. But according to Perez and Timble, half of the patients with IP of TLE present with schizophrenic psychosis. Since there is a danger of assuming that all psychoses of epilepsy are SCH, DSM 5 recommends a quantitative severity assessment of the primary symptoms.

The increased risk of psychiatric symptoms in epilepsy may be related to aspects that facilitate psychological manifestations, such as genetic loading and chronicity of TLE. The observation that transitory psychosis (postictal psychosis) may sometimes persist (as an interictal psychosis) depending on the patient's environmental or genetic background suggests a continuum of psychosis. It has been shown that healthy control participants with several degrees of schizotypal traits present with variable impaired frontotemporal connectivity. Based on the assumption that psychotic symptoms constitute a scale from absence of psychosis to full blown psychotic disorder, a similar scale of symptomatology could be built for psychotic manifestations of epilepsy. A possible continuum between psychosis and epilepsy has been suggested and hypothesized, explained by the kindling effect. The classical kindling process may gradually develop and spread through brain structures distal to the stimulated focus. The relatively long interval between the onset of TLE and development of psychosis suggest that damage to key structures is necessary and builds up over time.
There is controversy regarding the relationship between epilepsy and psychosis, but most agree that there is structural damage to limbic parts of the temporal lobe responsible for both epilepsy and psychosis. The discovery of common mechanisms or common affected neurotransmitters in TLE and SCH may disclose important clues about the vulnerability of TLE patients to develop psychosis and an opportunity to develop new treatment.

\section{Forced normalization: Antagonism between epilepsy and psychosis (3)}

The authors of this review article discuss the concept of forced normalization in epilepsy.

The term 'forced normalization' (FN) was first introduced by Heinrich Landolt, in the 1950s, to describe the emergence of behavioural and psychotic symptoms in patients with epilepsy, following normalization of their electroencephalographic abnormalities. The first monograph was released about 100 years before he coined the term. Hoffmann described a girl who developed paranoid psychosis, which was later found to be drug associated forced normalization. Samt later used the term 'transformed epilepsy' as he considered mental disorders and epilepsy to have the same diagnostic significance.

Wolf proposed possible mechanisms of FN and used the term 'paradoxical normalization'. His theory suggests that epilepsy remains active subcortically, and the concurrent activity of the inhibitory system spreads electroencephalographic discharges along uncertain pathways, inducing psychosis.

The exact neurobiological mechanism of FN is still not known. But several mechanisms are proposed in the literature, as described below.

\section{Electrical factors including kindling phenomenon}

Kindling was described by Alonso-DeFlorida and Delgado. The ability of kindling stimuli to produce behavioural changes has been demonstrated and this may explain the gradual development of psychosis in chronic epilepsy. The role of electroconvulsive therapy in the treatment for psychosis, and especially depression, is well documented. Psychosis secondary to vagal nerve stimulation have also been reported, suggesting that electrical stimulation of the brain plays at least a supporting role in the pathophysiology of FN.

\section{Resection}

The patients who underwent resection for epilepsy developed psychosis despite dramatic alleviation of epilepsy. This observation could not be explained by 
laterality, type of resection, histopathological diagnosis or total volume resected. However, there is no EEG evidence to support this theory.

\section{Chemical factors}

Some experiments have described behavioural responses when the limbic structures are activated selectively by using drugs, similar to what is seen in the limbic activation occurring during electrical kindling.

The role of neurotransmitters, especially dopamine and GABA have been studied in FN. Repeated administration of dopamine agonists and stimulants has been shown to produce behavioural responses, whereas dopamine antagonists are known to provoke seizures. Antiepileptics also have been implicated in FN.

The incidence of FN is reported to be $7.8 \%$ in adults with epilepsy. Most published reports on FN are limited to case presentations. The authors of this article conclude by mentioning that an increase in central dopamine activity by antiepileptics, may increase the risk of psychoses, and this needs further research with regard to FN.

\section{Association of depression and treated depression with epilepsy and seizure outcomes: A multicohort analysis (4)}

Community studies indicate that the prevalence of depression in patients with epilepsy is $23.1 \%$. A bidirectional relationship between the two conditions is suggested, with the possibility of shared pathophysiological mechanisms.

The authors of this study examined the hazard of depression among patients meeting the incident case definition for epilepsy, and explored whether those meeting the incident case definition of depression had an increased hazard of developing incident epilepsy. They also compared the hazard of depression following epilepsy and vice versa and examined whether depression was associated with worse seizure outcomes.

Data was extrapolated from a large UK database. Multiple logistic regression modeling was used to evaluate associations.

With regards to the results, when controlling for age, sex, and socioeconomic variables, the hazard of incident depression was higher for those with incident epilepsy compared to those who did not develop epilepsy.

When controlling for age, female sex and socioeconomic factors, the hazard of developing epilepsy was significantly higher for those developing incident depression compared to those free of depression. Furthermore, in the mediation analysis, the occurrence of depression influenced the relationship that gender, other comorbidities, socioeconomic factors, had on incident epilepsy, accounting for $4.6 \%, 7.1 \%$, and $20.6 \%$ of the total effects of these explanatory variables, respectively.

The presence of past or current depression was associated with higher odds of failing to achieve seizure freedom over the previous year compared with those free from depression. When focusing only on those with current or past depression, current treatment for depression treatment (antidepressants and/or counselling) was associated with a statistically significant higher odds of failing to achieve 1-year seizure freedom, after controlling for age at onset of epilepsy, sex, lesional epilepsy detected by magnetic resonance imaging, a history of generalized tonic-clonic seizures, and current antiepileptic drug use.

Although this study has its limitations, it suggests a temporal association and an apparent severity gradient in the association between depression and epilepsy. It provides evidence that depression partially mediates the effects of sex, socioeconomic factors, and other comorbidities on the risk of developing epilepsy. This suggests that depression-specific interventions might reduce the overall effect of each of these variables on epilepsy.

\section{Psychiatric comorbidities in epilepsy (5)}

Psychiatric comorbidities are common in epilepsy. There may be shared pathophysiological mechanisms that explain the association between epilepsy and the myriad of psychiatric comorbidities with which it is related. Recognition of these conditions is critical, and future research should aim to determine whether neuroanatomical mechanisms governing epilepsy-type (i.e., generalized vs focal epilepsy; temporal vs extra- temporal) are preferentially associated with specific psychiatric symptoms. Associated psychiatric comorbidities have a deleterious effect on quality-of-life, appear to portend worse seizure outcomes, and may be associated with medication non-adherence.

Psychiatric comorbidities in epilepsy are prone to being unrecognized and under-treated, thus leading to worse psychosocial and seizure-related outcomes. Prompt recognition and treatment of psychiatric comorbidities is paramount, since mood states highly influence qualityof-life. Furthermore, comorbid psychiatric conditions are associated with an increased risk of suicide and premature death. Therefore, persons with epilepsy should receive optimal therapy for their psychiatric comorbidities as well. 
Conflicts of interest

None declared

D Ariyasinghe, Department of Psychiatry, Faculty of M edicine, University Peradeniya

SR Perera, District General Hospital Nuwara Eliya

Corresponding author: SR Perera

Email: sayuperera@yahoo.com

http://orcid.org/0000-0001-6824-8991

\section{References}

1. Hill T, Coupland C, Morriss R, Arthur A, Moore M, Hippisley-Cox J. Antidepressant use and risk of epilepsy and seizures in people aged 20 to 64 years: A cohort study using a primary care database. BMC Psychiatry 2015; 15: 315 .

2. Kandratavicius L, Hallak JE, Leite JP. What are the similarities and differences between schizophrenia and schizophrenia-like psychosis of epilepsy? A neuropathological approach to the understanding of schizophrenia spectrum and epilepsy. Epilepsy Behav 2014; 38: 143-7.

3. Kawakami Y, Itoh Y. Forced normalization: Antagonism between epilepsy and psychosis. Pediatr Neurol 2017; 70: 16-9.

4. Josephson CB, Lowerison M, Vallerand I, Sajobi TT, Patten S, Jette N, Wiebe S. Association of depression and treated depression with epilepsy and seizure outcomes: A multicohort analysis. JAMA Neurol 2017; 74(5): 533-9.

5. Josephson CB, Jetté N. Psychiatric comorbidities in epilepsy. Int Rev Psychiatry 2017; 29(5): 409-2424. 